\title{
Origin of Ultrafast Component of Photoluminescence Decay in Nanostructures Doped with Transition Metal or Rare-Earth Ions
}

M. GOdLEWSKi ${ }^{a, b}$, S. YATSUnenKo ${ }^{a}$, V.Yu. IVAnOV ${ }^{a}$, A. Khachapuridze ${ }^{a}$, K. ŚWiA̧TEK ${ }^{a}$, E.M. GOLDYS ${ }^{c}$, M.R. Phillips ${ }^{d}$, P.J. Klar ${ }^{e}$ AND W. Heimbrodt ${ }^{e}$

${ }^{a}$ Institute of Physics, Polish Academy of Sciences al. Lotników 32/46, 02-668 Warsaw, Poland

${ }^{b}$ Dept. of Mathematics and Natural Sciences, College of Science

Cardinal S. Wyszyński University, Warsaw, Poland

${ }^{c}$ Div. of Information and Communication Sciences, Macquarie Univ. Sydney, Australia

${ }^{d}$ Microstructural Analysis Unit, UTS, Sydney, Australia

${ }^{e}$ Dept. of Physics and Materials Sciences Center, Phillips-University of Marburg

Renthof 5, 35032 Marburg, Germany

Bulk samples, layers, quantum well, and quantum dot structures of II-Mn-VI samples all show coexistence of slow and fast components of $\mathrm{Mn}^{2+}$ photoluminescence decay. Thus, fast photoluminescence decay cannot be related to low dimensionality of a host material. This also means that the model of the so-called quantum confined atom is incorrect. Based on the results of time-resolved photoluminescence and optically detected magnetic resonance investigations we relate the observed lifetime decrease in $\mathrm{Mn}^{2+}$ intra-shell transition to spin dependent magnetic interactions between localized spins of $\mathrm{Mn}^{2+}$ ions and between $\mathrm{Mn}^{2+}$ ions and spins/magnetic moments of free carriers. The latter mechanism is enhanced in nanostructures.

PACS numbers: 71.55.Gs, 76.30.Fc, 76.70.Hb, 78.55.Et

\section{Introduction}

${ }^{4} T_{1}$-to- ${ }^{6} A_{1}$ transition of $\mathrm{Mn}^{2+}$ ions in wide band gap II-VI semiconductors is parity and spin forbidden process. In the consequence, very long (in the range of $\mu \mathrm{s}-\mathrm{ms}$ ) photoluminescence (PL) decay times were observed $[1,2]$. Surprisingly, 
Bhargava and co-workers reported a dramatic shortening of PL lifetime of $\mathrm{Mn}^{2+}$ intra-shell transition for ZnMnS nanoparticles [3, 4]. Significant shortening of the PL decay time (by six orders in magnitude) was observed together with a high quantum efficiency of PL (of approximately 20\%), i.e., shortening of PL lifetime could not be related to competition of some nonradiative processes. The effect was related to a quantum confinement on $\mathrm{Mn}^{2+}$ ions (model of quantum confined atom) in nanostructures of wide band gap II-Mn-VI compounds [3, 4]. In this model, quantum confinement enhances $s p-d$ hybridization and thus relaxes parity and spin selection rules for Mn intra-shell transition [3, 4]. Similar enhancement of recombination rate was claimed to be present in the case of nanostructures activated with rare-earth ( $\mathrm{RE})$ ions [5].

Dramatic shortening of lifetime of ${ }^{4} T_{1}$-to- ${ }^{6} A_{1}$ intra-shell transition was unexpected and difficult, or saying more precisely, impossible to explain if one relies on a standard crystal-field model. In fact, all our attempts, which were based on a calculation scheme proposed by Boulanger et al. [6, 7], indicated that spin-spin interactions (cross-relaxation) between adjacent Mn ions, or Mn ions and free carriers can result in PL lifetime shortening by at most three orders in magnitude [8], but not by six orders, as reported by Bhargava and co-workers [3, 4]. The spinorbit interaction, magnetic dipole and exchange interactions or the one with the radiative field are not strong enough to relax sufficiently spin and parity selection rules for ${ }^{4} T_{1}$-to- ${ }^{6} A_{1}$ intra-shell transition of $\mathrm{Mn}^{2+}$ ions. This conclusion was made by Kuo Yan et al., too [9].

Not surprisingly Bol and Meijerink claimed that the shortening of the PL lifetime is an experimental artifact [10]. Bol and Meijerink [10], and also the present authors [11], have shown that PL of $\mathrm{Mn}^{2+}$ in nanostructures shows "normal" $\mathrm{ms}$ range $\mathrm{PL}$ decay, observed together with a fast component of the $\mathrm{PL}$ decay, in contrast to the predictions of the quantum confinement model. Bol and Meijerink related ultrafast component of the PL decay to another PL, which overlaps with $\mathrm{Mn}^{2+}$ intra-shell PL [10]. Thus, in their model fast PL decay is not an internal feature of $\mathrm{Mn}^{2+} \mathrm{PL}$. We argue with such interpretation and relate the fast component of the PL decay to $\mathrm{Mn}^{2+}$ intra-shell transition. The relevant mechanisms responsible for fast PL decay are discussed in this work.

Kuo Yan et al. [9] proposed another explanation of ultrafast PL decay. They assumed formation of coupled stationary states of Mn-host with $S=5 / 2$ for $\mathrm{Mn}^{2+}$ ions and $s=1 / 2$ for free electrons. In the consequence, the total spin of a coupled state is either $S=2$ or $S=3$ state, depending on a relative spin orientation of two coupled states. In their model strong exchange interaction between $d$ electrons and a coupled host electron relaxes spin selection rules for ${ }^{4} T_{1}$-to- ${ }^{6} A_{1}$ intra-shell transition, by flipping spin of a coupled single-electron state. These authors indicated that their model is clearly low-dimensionality related, since the mentioned coupled states are formed due to a strong overlap of electron and Mn wave function, expected in nanoparticles. 
The above model can explain why spin selection rules are relaxed for Mn intra-shell transition. It remains, however, an open question if the mechanism proposed is sufficiently efficient to account for the results of Bhargava et al. The recent estimations performed by Huong and Birman [12] indicate that the above-mentioned mechanism can shorten PL lifetime by at most three orders in magnitude, and not by six. Moreover, as we will show further on, shortening of the PL lifetime is not a unique feature of low-dimensional systems. Another weak point of the model of Kuo Yan et al. is that electron spin resonance (ESR) spectra of Mn doped nanoparticles are fairly similar to those of Mn doped bulk samples, as discussed below and based on our ESR results. Thus, we do not agree with the statement of Kuo Yan et al. [9] that ESR of nanoparticles supports their model.

To clarify all the above-mentioned models we performed PL, PL kinetics, ESR, and optically detected magnetic resonance (ODMR) investigations on several nanostructures, quantum dot (QD), quantum well (QW), and bulk samples of II-Mn-VI compounds. First, we shall demonstrate that the fast component of the PL decay is not low-dimensionality related. We then estimate efficiency of spin-flip interactions between Mn ions and between Mn ions and spins (magnetic moments) of free carriers. Both these interactions can partly relax spin selection rules for Mn intra-shell transitions, i.e., can increase PL recombination rate, as is demonstrated experimentally. Next, the origin of ultrafast component of the PL decay is elucidated, based on the PL kinetics investigations of CdMnTe QD sample.

\section{Experimental}

The experiments were performed for a range of II-Mn-VI materials of a different dimensionality and with different $\mathrm{Mn}$ fractions: the nanostructures of $\mathrm{ZnMnS}$ and $\mathrm{CdMnS}$ synthesized in the mesoporous MCM-41 $\mathrm{SiO}_{2}$ matrices [13] with grains of 5-40 nm size as estimated in electron microscopy; ZnMnS, ZnMnO, ZnMnSe, and CdMnTe bulk samples; self-organized CdMnTe QDs grown by molecular beam epitaxy on ZnTe, and QW structures of CdMnTe/CdMgTe. Stationary PL, time-resolved PL, ESR, and ODMR setups used in the present study are described elsewhere $[8,11,14,15]$.

\section{Results and discussion}

As already mentioned, Bol and Meijerink [10] related fast component of the PL decay in nanostructures of $\mathrm{ZnMnS}$ to an underlying PL of a different origin. We tested several II-Mn-VI systems of a different dimensionality to check such possibility. Based on these investigations we claim that the fast component of PL decay is related to $\mathrm{Mn}^{2+}$ intra-shell transition. Thus the following discussion concentrates on search of relevant mechanism(s) responsible for lifetime shortening. 
First of all we checked whether $\mathrm{Mn}^{2+}$ ions are present in our ZnMnS and CdMnS nanostructures, by performing ESR experiments. Strong magnetic resonance of $\mathrm{Mn}^{2+}$ was observed showing well-resolved six-line hyperfine (HF) structure. This structure is typically not resolved for increased Mn fractions (above $0.5 \%$ ) in bulk samples [16]. For nanostructures, however, the HF structure was resolved even for nanopowders with $30 \% \mathrm{Mn}$ fraction. For $\mathrm{ZnMnS}$ nanopowders two overlapping ESR signals were observed - the first shows a characteristic 6-line HF structure, the second consists of a broad and featureless line with the same $g$-factor. We relate the former signal to isolated $\mathrm{Mn}^{2+}$ ions, most likely located close to surface of nanopowders. The latter signal most likely comes from "body" of nanopowders. There, due to increased number of Mn neighbors, the HF structure is broadened by spin-spin interactions between adjacent Mn ions, and thus is not resolved.

Microwave power and temperature dependences of the signals were studied. Only in the case of bulk ZnMnSe sample with a relatively low Mn fraction (about $0.5 \%$ ) the ESR signal saturated quickly with an increase in microwave power. In most of other cases, in particular for nanopowders studied by us, ESR signal increased with increase in microwave power, indicating a significant shortening of spin relaxation time. We directly confirmed this observation using time-resolved ODMR, observing fast spin relaxation times, in particular upon photoexcitation [14, 15].

Next we studied optical properties of II-Mn-VI samples. Below we concentrate on discussion of optical properties of $\mathrm{ZnMnS}$ samples. Results obtained for other samples are only used if necessary to demonstrate relevant interactions.

Figure 1 shows the PL spectrum related to ${ }^{4} T_{1}$-to- ${ }^{6} A_{1}$ transition of $\mathrm{Mn}^{2+}$ in ZnMnS. The PL spectra observed for bulk ZnMnS and for ZnMnS nanoparticles, both with $1 \% \mathrm{Mn}$ fraction, are identical. In both cases the PL intensity is enhanced under a direct excitation into one of excited intra-shell states of $\mathrm{Mn}^{2+}$ ion (e.g. for $514 \mathrm{~nm}$ excitation). This enhancement is more pronounced in nanoparticles. $514 \mathrm{~nm}$ excitation induces also a low energy wing of the PL of nanoparticles (not shown in Fig. 1). An origin of this low energy PL wing, extending down to $1.6 \mathrm{eV}$, remains unknown. It can be due to some underlying PL of a different origin, or due to PL emission of $\mathrm{Mn}$ ions in a different environment. The latter can be due to different spectral responses of isolated $\mathrm{Mn}^{2+}$ ions and $\mathrm{Mn}^{2+}$ associates, complexes, etc.

We then checked if fast components of the PL decay are features of low-dimensionality of a given II-Mn-VI sample. PL decay kinetics was measured by setting detection at the PL maximum and using two excitation conditions, i.e., the above band gap $(266 \mathrm{~nm})$ and the intra-shell $(514 \mathrm{~nm})$ excitation. The relevant data are shown in Figs. 2 and 3. For nanoparticles PL decay (see Fig. 2) shows a very pronounced (see time scale) fast component of the decay, which is observed together with a "normal" slow decay component in the ms range (not 


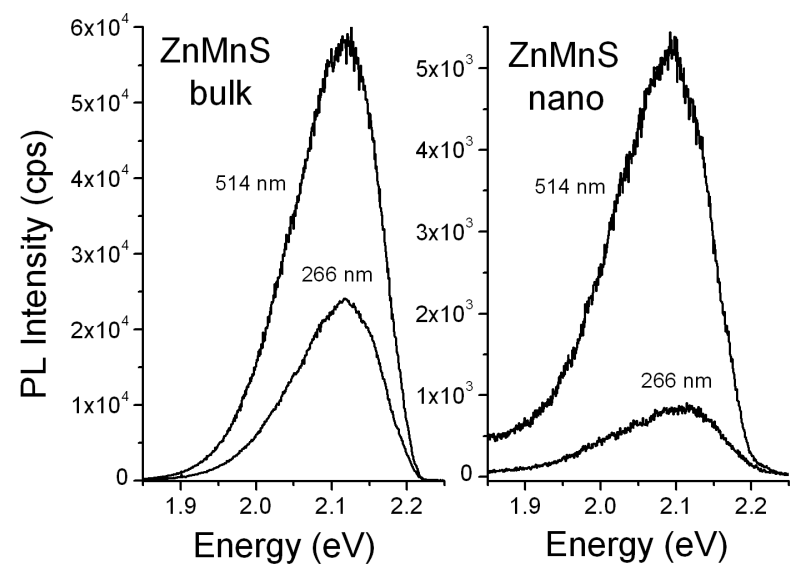

Fig. 1. Low temperature photoluminescence spectra of (left part) bulk ZnMnS and (right part) ZnMnS nanostructures (both with $1 \%$ of Mn), measured at two excitation conditions, at the above band gap excitation $(266 \mathrm{~nm})$ and at intra-shell excitation $(514 \mathrm{~nm})$.

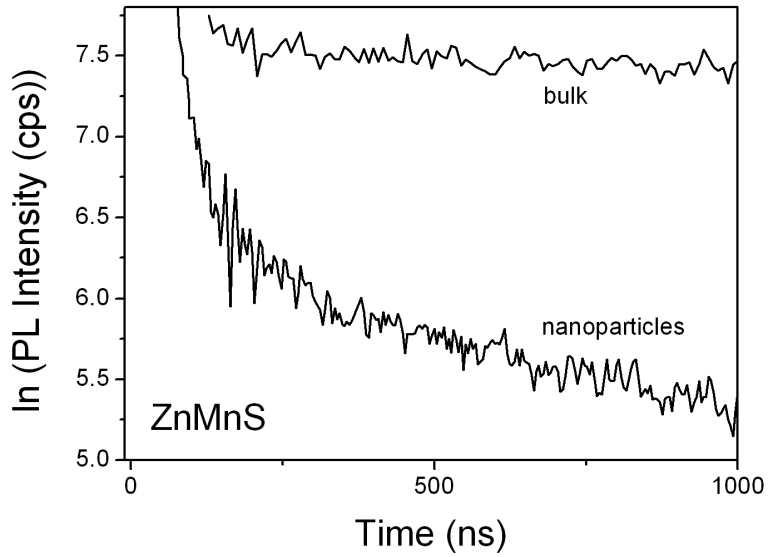

Fig. 2. Comparison of PL kinetics of $\mathrm{Mn}^{2+}$ intra-shell emission in nanostructures of $\mathrm{ZnMnS}$ and in bulk ZnMnS (Mn fraction of 1\%) measured at the above band gap excitation $(266 \mathrm{~nm})$.

shown in Fig. 2). The component with decay time of 50 microseconds dominates. Fast component of the PL decay is also observed for bulk $\mathrm{ZnMnS}$ ( $1 \%$ of $\mathrm{Mn}$ ) sample (Fig. 3). The contribution of this component in the PL decay is enhanced at the above band gap excitation, as shown in Fig. 3. We obtained similar results for all II-Mn-VI materials studied. In all cases (for samples of different dimensionality) fast and slow components of the PL decay coexist. We thus conclude that the fast component of the PL decay cannot be a unique feature related to low-dimensionality of the system. 


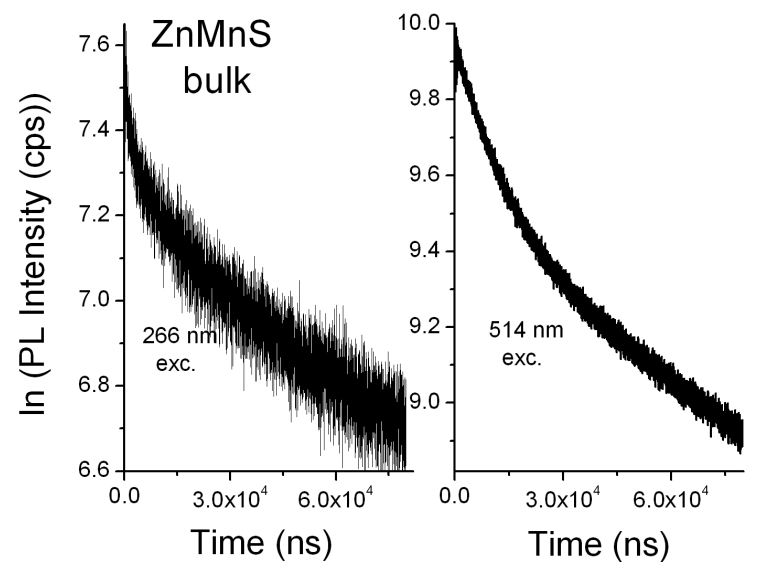

Fig. 3. PL kinetics of $\mathrm{Mn}^{2+}$ intra-shell emission in bulk $\mathrm{ZnMnS}$ (Mn fraction of 1\%) measured at two excitation conditions, at the above band gap excitation (266 nm, left part) and at intra-shell excitation (514 nm, right part).

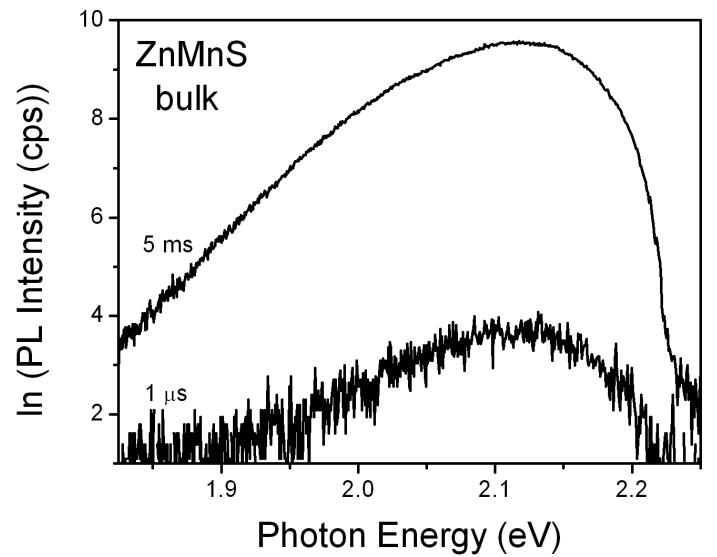

Fig. 4. Time-resolved PL of $\mathrm{Mn}^{2+}$ intra-shell emission in bulk $\mathrm{ZnMnS}$ (Mn fraction of $1 \%$ ) measured at the above band gap excitation $(266 \mathrm{~nm})$ and at two time intervals (window width) after photoexcitation.

To evaluate contribution of sub-microsecond component of the PL decay we employed time-resolved PL. Time integrated PL spectra were measured by varying window size followed by the above band gap excitation. In the experiment we measured a number of excited $\mathrm{Mn}^{2+}$ ions emitting light in a set time window, following photoexcitation. In Fig. 4 we show results of such investigations for bulk ZnMnS sample (1\% of Mn). Figure 4 compares (logarithmic scale is used) PL spectra measured in such way for 1 microsecond and 5 millisecond widths of time windows. Sub-microseconds component in the PL decay is present, but is not efficient. For bulk ZnMnS sample only small percentage of the Mn ions decays 
during the first microsecond after the excitation. Similar investigations, and also data shown in Fig. 2, show that for nanoparticles the process(es) responsible for the fast component of the PL decay is (are) more efficient. Fast component of the PL decay is enhanced in samples of low-dimensionality.

We applied ODMR to identify mechanism(s) responsible for shortening of decay time of $\mathrm{Mn}^{2+} \mathrm{PL}[11,14,15,17]$. In this study $\mathrm{Mn}^{2+}$ magnetic resonance was detected optically. $\mathrm{Mn}^{2+}$ resonance with a well-resolved HF structure (6 lines) was observed for ZnMnSe with $\mathrm{Mn}$ fraction about $0.5 \%$, allowing us an unambiguous identification of the magnetic resonance signal. In the case of bulk $\mathrm{ZnMnS}$ (1\% of $\mathrm{Mn}$ ) only wide resonance was detected, identical to the one observed in the ESR study.

We measured spectral response (the so-called ODMR-PL spectrum) of the resonance to identify the relevant recombination mechanisms. We found that $\mathrm{Mn}^{2+}$ resonance is detected via an increase in intensity of $\mathrm{Mn}^{2+} \mathrm{PL}$. This observation means that flipping of a spin of $\mathrm{Mn}^{2+}$ in the ${ }^{6} A_{1}$ ground state (induced in magnetic resonance) shortens PL decay time of a nearby excited $\mathrm{Mn}^{2+}$ ion in the ${ }^{4} T_{1}$ state. Spin cross-relaxation interactions between Mn ions must thus be relatively efficient and must affect PL recombination rate.

The conclusion about the efficiency of such interactions is supported by the ESR investigations discussed above. In ESR we observed shortening of spin relaxation times in samples with increased Mn fractions. For such samples saturation of the $\mathrm{Mn}^{2+}$ ESR signal was not observed even at large microwave powers. Such saturation is commonly observed for samples lightly doped with Mn. The effect is more pronounced in nanopowders.

Even though Mn-Mn spin flip interactions (cross-relaxation) can reduce PL decay time, ODMR investigations as well as theoretical estimations [12] indicate that these interactions cannot account for the ultrafast component of the PL decay observed by Bhargava and co-workers. In ODMR-PL we observed that $\mathrm{Mn}-\mathrm{Mn}$ cross-relaxation increases PL decay rate by a few percent only, which explains why in all cases studied by us a slow component of the PL decay (in ms time scale) is co-present and, moreover, often dominates the PL decay spectra.

Stationary PL, PL kinetics, and ODMR investigations indicate that different spin-flip interactions can be more efficient and can significantly contribute to the observed shortening of the PL decay time. For example, ODMR investigations of CdMnTe QW system indicate that spin relaxation rate depends on excitation intensity if free carriers are photoexcited [11, 14, 15], suggesting that the spin-flip interactions with free carriers can significantly affect rate of $\mathrm{Mn}^{2+} \mathrm{PL}$. Also, comparison of the PL kinetics of the $\mathrm{Mn}^{2+}$ PL measured at two excitation conditions (Figs. 2 and 3) suggests similar effect. Fast component of the $\mathrm{Mn}^{2+} \mathrm{PL}$ decay is enhanced at the above band gap excitation. Co-excitation of free carriers results in shortening of the PL decay time. 

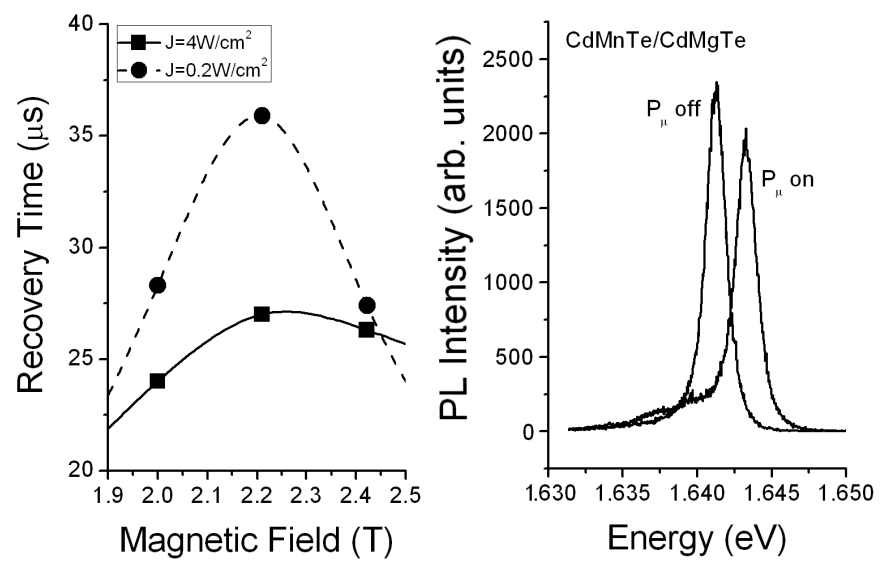

Fig. 5. PL shift of the excitonic emission from $8 \mathrm{~nm}$ wide CdMnTe (1\% of Mn) quantum well in CdMnTe/CdMgTe (20\% of Mg) structure measured at magnetic field set at $\mathrm{Mn}^{2+}$ magnetic resonance conditions (right) and recovery time of sample magnetization after turning off microwave power (left).

Following this idea, we directly evaluated influence of photoexcitation density on spin relaxation times, applying time-resolved ODMR study (Fig. 5). To explain the latter experiment we need a short introduction to the properties of II-Mn-VI systems. An external magnetic field aligns spins of magnetic impurities, inducing magnetization in the sample [16]. The Zeeman splitting observed for excitonic transitions is then proportional to the sample magnetization, and is exceptionally large.

Sample magnetization (and thus Zeeman splitting) can be reduced at $\mathrm{Mn}^{2+}$ magnetic resonance in the ODMR study. The effect is large in the case of slow spin relaxation rates, and is small, if spin relaxation is fast. The magnitude of the effect can be studied by observing shift in a spectral position of a relevant excitonic emission (see Fig. 5). If magnetization is reduced, the Zeeman splitting is also reduced. The PL shifts up in energy in this case, as seen in Fig. 5 (right part). We found that the magnitude of this shift depends on excitation conditions. By increasing photoexcitation density we could significantly decrease PL shift, which we relate to a significant increase in spin relaxation rate of $\mathrm{Mn}^{2+}$ ions.

We used this fact to determine spin relaxation times in our system, by performing time-resolved ODMR investigations. We measured "recovery time" of the sample magnetization after turning off microwave power at $\mathrm{Mn}^{2+}$ magnetic resonance (Fig. 5, left part). We first set the magnetic field at $\mathrm{Mn}^{2+}$ magnetic resonance (at about $2.2 \mathrm{~T}$ for our $60 \mathrm{GHz}$ ODMR system) and then measured the time needed for the PL to shift up (microwaves on) and down (microwaves off) in energy. In these experiments we used CdMnTe/CdMgTe single QW sample for which sharp, well-resolved excitonic emission was observed, allowing precise 


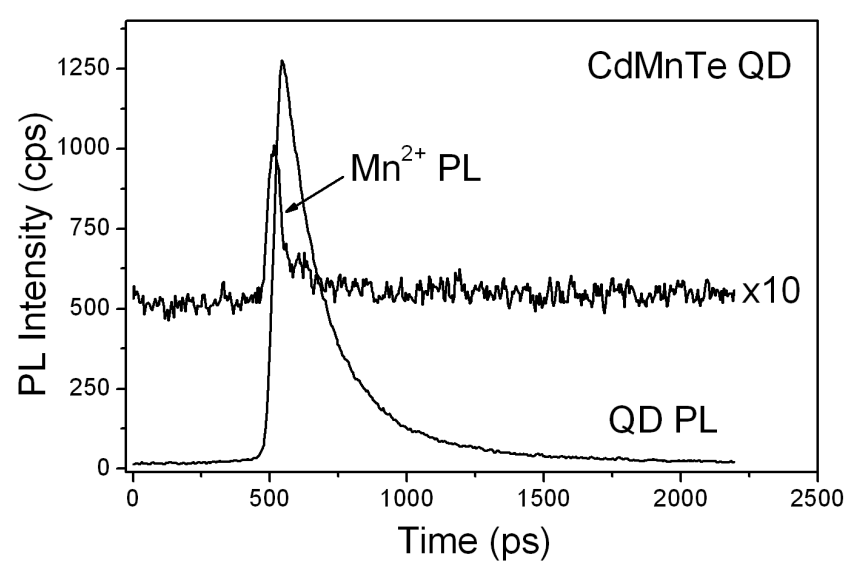

Fig. 6. Comparison of PL kinetics of $\mathrm{Mn}^{2+}$ intra-shell emission and of $\mathrm{QD}$ excitonic emissions in self-organized CdMnTe quantum dots.

measurements of a spectral shift of the PL line. The so-estimated spin relaxation time is about $37 \mu$ s at low excitation density and shortens to $27 \mu$ s at increased excitation density.

All performed by us investigations indicate that spin-flip interactions between the localized spins of $\mathrm{Mn}$ ions and spins/magnetic moments of free carriers are efficient, especially for interactions with the free holes [17]. Theoretical evaluation suggests, however, that such process is not efficient enough to account for observation of Bhargava et al. [12].

Even though the estimated shortening of spin relaxation times is inadequate, the possible explanation of the ultrafast component of the PL decay relates to this interaction. This claim is based on the results of PL kinetics measurements performed for CdMnTe QD system [11, 15]. Comparing rise kinetics of the excitonic PL and decay kinetics of $\mathrm{Mn}^{2+}$ intra-shell PL we noticed (see Fig. 6) that the shortest component of the $\mathrm{Mn}^{2+} \mathrm{PL}$ decay reflects lifetime of free carriers in a given sample. The ultrafast component of the PL decay is thus a measure of trapping time of free carriers into excitons, trapping by deep centers, etc. Once free carriers are trapped, rate of PL decay is reduced, explaining observed drop of PL intensity. This observation confirms our conclusion on efficient spin-flip interactions between Mn ions and free carriers.

\section{Conclusions}

In conclusion, we have demonstrated that the fast component of the $\mathrm{Mn}^{2+}$ PL decay is present both in low-dimensional structures and in bulk samples. This effect is thus not related to quantum confinement of $\mathrm{Mn}^{2+}$ ions, but is only enhanced in low-dimensional structures. We further demonstrate that the spin-flip interactions between localized spins of $\mathrm{Mn}^{2+}$ ions and between $\mathrm{Mn}^{2+}$ ions and spins 
of free carriers can efficiently relax selection rules for $\mathrm{Mn}^{2+}$ intra-shell transition. We also expect that similar mechanism is active in RE doped nanostructures, explaining origin of the PL decay shortening. Both effects can reduce PL decay time by one-to-three orders in magnitude, but not to six orders. The ultrafast component of the PL decay relates in our opinion to a lifetime of free carriers in a given system.

\section{Acknowledgments}

This work was partly supported by the grant no. PBZ-KBN-044/P03/2001 of the State Committee for Scientific Research (Poland).

\section{References}

[1] W. Busse, H.-E. Gumlich, B. Meissner, D. Theis, J. Lumin. 12/13, 693 (1976).

[2] V.F. Agekyan, Phys. Solid State 44, 2013 (2002).

[3] R.N. Bhargava, J. Lumin. 70, 85 (1996).

[4] R.N. Bhargava, D. Gallagher, Phys. Rev. Lett. 72, 416 (1994).

[5] R.N. Bhargava, V. Chhabra, B. Kulkarni, A. Ekimov, N. Taskar, A. Mehta, M. Barnes, T. Thundat, in: Phys. Semicond. 2002, Proc. 26th Int. Conf. on the Phys. Semicond., Inst. of Phys. Conf. Series No. 171, Eds. A.R. Long, J.H. Davies, paper Q3.5.

[6] D. Boulanger, R. Parrot, J. Chem. Phys. 91, 5500 (1989).

[7] D. Boulanger, D. Curie, R. Parrot, J. Lumin. 48/49, 680 (1991).

[8] V.Yu. Ivanov, M. Godlewski, S. Yatsunenko, A. Khachapuridze, M.S. Li, Z. Gołacki, Acta Phys. Pol. A 100, 351 (2001).

[9] Kuo Yan, Chnagkui Duan, Yi Ma, Shanda Xia, J.-C. Krupa, Phys. Rev. B 58, 13585 (1998).

[10] A.A. Bol, A. Meijerink, Phys. Rev. B 58, R15997 (1998).

[11] M. Godlewski, V.Yu. Ivanov, P.J. Bergman, B. Monemar, Z. Gołacki, G. Karczewski, J. Alloys Comp. 341, 8 (2002).

[12] Nguyen Que Huong, J.L. Birman, Phys. Rev. B 69, 085321 (2004).

[13] L. Chen, P.J. Klar, W. Heimbrodt, F. Brieler, M. Fröba, Appl. Phys. Lett. 76, 3531 (2000).

[14] M. Godlewski, V.Yu. Ivanov, A. Khachapuridze, S. Yatsunenko, Phys. Status Solidi B 229, 533 (2002).

[15] M. Godlewski, S. Yatsunenko, A. Khachapuridze, V.Yu. Ivanov, Z. Gołacki, G. Karczewski, P.J. Bergman, P.J. Klar, W. Heimbrodt, M.R. Phillips, J. Alloys Comp. 380, 45 (2004).

[16] J.K. Furdyna, J. Appl. Phys. 64, R29 (1988).

[17] M. Godlewski, A. Wittlin, R.R. Gałązka, B. Monemar, T. Gregorkiewicz, C.A.J. Ammerlaan, P.H.M. van Loosdrecht, J.A.A.J. Perenboom, in: The Physics of Semiconductors, Proc. 23rd Int. Conf. ICPS'1996, Berlin 1996, Eds. M. Scheffler, R. Zimmermann, World Scientific, Singapore 1996, Vol. 1, p. 393. 